D. Barron ${ }^{a}$. P. Ade Ad A. Anthony $^{c} \cdot$ K.

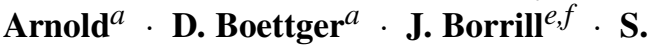

Chapman $^{n} \cdot$ Y. Chinone ${ }^{g} \cdot$ M. Dobbs ${ }^{h} \cdot$ J.

Edwards $^{a}$. J. Errard ${ }^{e}$. G. Fabbian ${ }^{i}$. D.

Flanigan $^{d}$. G. Fuller ${ }^{a}$. A. Ghribi ${ }^{d}$. W.

Grainger $^{O}$. N. Halverson ${ }^{c}$. M. Hasegawa ${ }^{g}$.

K. Hattori ${ }^{g}$ - M. Hazumi ${ }^{g}$. W. Holzapfel ${ }^{d}$.

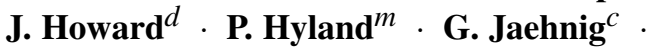

A. Jaffe ${ }^{j} \cdot$ B. Keating ${ }^{a}$. Z. Kermish ${ }^{p} \cdot$ R.

Keskitalo $^{e} \cdot$ T. Kisner ${ }^{e} \cdot$ A. T. Lee ${ }^{d} \cdot$ M.

Le Jeune $^{i} \cdot$ E. Linder ${ }^{k}$. M. Lungu ${ }^{d} \cdot \mathbf{F}$.

Matsuda $^{a}$. T. Matsumura ${ }^{g}$. X. Meng ${ }^{a}$. N.

J. Miller $^{q}$ - H. Morii ${ }^{g}$. S. Moyerman ${ }^{a}$. M.

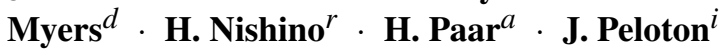

- E. Quealy ${ }^{d}$. G. Rebeiz ${ }^{a}$. C. L. Reichardt ${ }^{d}$

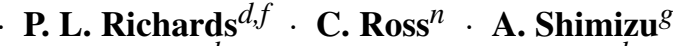

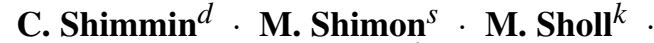

P. Siritanasak ${ }^{a}$. H. Spieler ${ }^{k}$. N. Stebor ${ }^{a}$.

B. Steinbach ${ }^{d}$. R. Stompor ${ }^{i}$. A. Suzuki St $^{d}$.

T. Tomaru ${ }^{g}$. C. Tucker ${ }^{b}$. A. Yadav ${ }^{a}$. O.

Zahn $^{k, l}$

\title{
The POLARBEAR Cosmic Microwave Background Polarization Experiment
}

07.15 .2013

\footnotetext{
${ }^{a}$ Physics Department, University of California, San Diego; E-mail: dbarron@physics.ucsd.edu

$b_{\text {School of Physics and Astronomy, University of Cardiff; }}$

${ }^{c}$ Department of Astrophysical and Planetary Science, University of Colorado;

$d_{\text {Physics Department, University of California, Berkeley; }}$

${ }^{e}$ Computational Cosmology Center, Lawrence Berkeley National Laboratory;

$f_{\text {Space Sciences Laboratory, University of California, Berkeley; }}$

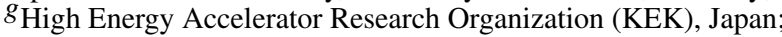

$h_{\text {Physics Department, McGill University; }}$

${ }^{i}$ Laboratoire Astroparticule et Cosmologie (APC), Universite Paris 7;

$j_{\text {Department of Physics, Imperial College; }}$

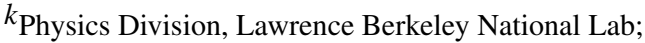

${ }^{l}$ Berkeley Center for Cosmological Physics (BCCP), University of California, Berkeley;

$m_{\text {Physics Department, Austin College; }}$
} 


\begin{abstract}
The POLARBear Cosmic Microwave Background (CMB) polarization experiment has been observing since early 2012 from its 5200 meter site in the Atacama Desert in Northern Chile. POLARBEAR's measurements will characterize the expected $\mathrm{CMB}$ polarization due to gravitational lensing by large scale structure, and search for the possible B-mode polarization signature of inflationary gravitational waves. POLARBEAR's $250 \mathrm{mK}$ focal plane detector array consists of 1,274 polarization-sensitive antenna-coupled bolometers, each with an associated lithographed band-defining filter and contacting dielectric lenslet, an architecture unique in current CMB experiments. The status of the POLARBEAR instrument, its focal plane, and the analysis of its measurements are presented.
\end{abstract}

Keywords cosmic microwave background, CMB polarization, millimeter-wave

\title{
1 Introduction
}

From its discovery to the present, a series of more detailed measurements of the cosmic microwave background, including its primary temperature anisotropy and E-mode (curl-free) polarization, have helped refine our models of the universe ${ }^{2,3}$. Many current CMB experiments, including POLARBEAR, are focused on characterizing the B-mode (divergence-free) polarization component of the CMB.

At large angular scales, B-mode polarization is predicted to have been generated by primordial gravitational wave tensor perturbations during inflation ${ }^{1}$. The strength of these tensor perturbations is dependent on the shape of the inflationary potential as well as the energy scale of inflation, but it is expected to peak on an angular scale of 2 degrees. Detection of this B-mode polarization would be direct evidence supporting the inflationary paradigm, and would help to constrain the parameter space of inflationary models. ${ }^{5}$.

Although tensor perturbations are the only primordial source of B-mode polarization, B-modes are also created at later times by weak gravitational lensing of the CMB by large scale structure. This lensing mixes E and B-modes, creating B-mode polarization that has recently been detected on small angular scales ${ }^{4}$. Lensing B-modes can give information about the large scale structure that generated them, as the lensing effect is sensitive to the formation of structure at early epochs. POLARBEAR's observation fields overlap with optical and infrared galaxy surveys, and cross-correlation with these data sets will leverage their redshift information for a more complete picture of the lensing effect.

\section{Instrument Overview}

Reaching the sensitivity necessary to measure the CMB's B-mode polarization requires significant advances in detector technology. POLARBEAR uses a unique

\footnotetext{
${ }^{n}$ Physics Department, Dalhousie University;

${ }^{o}$ Rutherford Appleton Laboratory, STFC;

$p_{\text {Princeton University; }}$

$q_{\text {NASA Goddard Space Flight Center; }}$

$r_{\text {Kavli Institute for the Physics and Mathematics of the Universe, University of Tokyo; }}$

${ }^{s}$ Tel Aviv University
} 


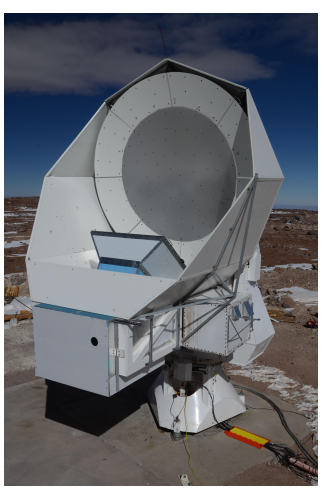

(a)

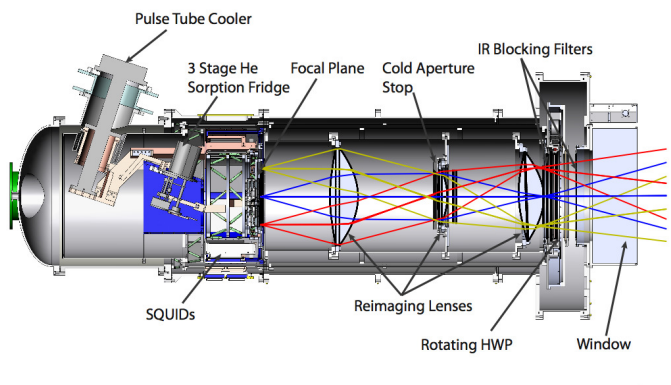

(b)

Fig. 1: (a) The Huan Tran Telescope (b) A cross-sectional drawing of the POLARBEAR cryogenic receiver with major components identified. (Color figure online)

637 pixel lenslet-coupled focal plane, integrated with a large field of view telescope and cold reimaging optics, and observing in a single $38 \mathrm{GHz}$ wide spectral band centered at $148 \mathrm{GHz}$. This section gives a brief overview of the instrument. The design and development of the POLARBEAR experiment have been described in detail in previous proceedings $6,7,8$.

POLARBEAR is mounted on the Huan Tran Telescope (HTT), built by VertexRSI ${ }^{i}$, which is an off-axis Gregorian design that satisfies the Mizuguchi-Dragone condition. This optical design has a large diffraction limited field of view of 2.3 degrees, along with low sidelobe response and low cross polarization, meeting the systematic requirements for POLARBEAR's science goals. ${ }^{9,10}$. The primary mirror is a 2.5 meter monolithic piece of cast aluminum, precision machined to $53 \mu \mathrm{m}$ rms surface accuracy, with a lower-precision guard ring extending to 3.5 meter diameter. The primary produces a 3.5 arcminute FWHM beam at $148 \mathrm{GHz}$. The secondary mirror is $1.4 \mathrm{~m}$, with baffling enclosing it and the receiver window to block scattered light.

The transition edge sensor (TES) detectors are designed to operate at 0.25 Kelvin, where thermal carrier noise becomes subdominant compared to expected thermal background loading noise from the Chilean atmosphere. The bolometers have a design noise equivalent temperature (NET) of $500 \mu \mathrm{K}_{C M B} \sqrt{s}$. The cryogenic receiver, shown in Figure 1b, has a cumulative optical efficiency of $37 \%$, including contributions from the focal plane, aperture stop, lenses, and filters ${ }^{6}$. A two-stage pulse tube refrigerator ${ }^{\mathrm{ii}}$ providing continuous cooling power at 50 Kelvin and 4 Kelvin. A three-stage helium sorption fridge ${ }^{\mathrm{iii}}$ provides two cooling stages at 0.35 Kelvin and 0.25 Kelvin, with a hold time greater than 30 hours.

The focal plane, shown in Figure 2a, consists of seven modular arrays of antenna-coupled transition edge sensor (TES) detectors, each with 192 detectors. An individual pixel, shown in Figure 2b, consists of two Al/Ti bilayer TES detec-

\footnotetext{
i http://www.gdsatcom.com/vertexrsi.php

ii http://www.cryomech.com

iii http://www.chasecryogenics.com
} 


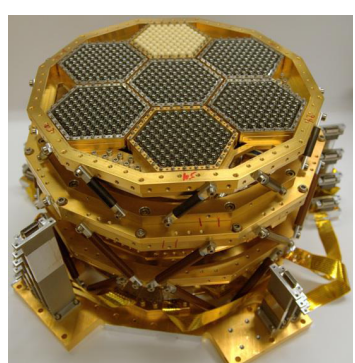

(a)

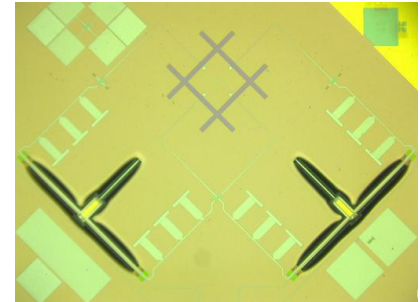

(b)

Fig. 2: (a) POLARBEAR's complete focal plane including lenslets, support structures and wiring. One array module has white alumina instead of silicon lenslets (b) A single pixel, with two TES detectors and slot antenna (Color figure online)

tors, coupled to orthogonal polarizations of the dual-polarized slot antenna, with on-chip band-defining filters ${ }^{7}$. Each pixel is paired with a beam-forming, antireflection coated lenslet ${ }^{11}$. Fluctuations in optical power are converted to changes in current in the voltage-biased TES detector, and this current is read out using superconducting quantum interference devices (SQUIDs). Reading out large arrays of detectors requires signal multiplexing in order to reduce thermal loading on the cold focal plane, as well as to reduce the cost, size, and complexity of cryogenic wiring and other cold readout components. POLARBEAR uses frequency-domain multiplexing ${ }^{12}$ with a multiplexing factor of eight.

\section{Instrument Performance}

POLARBEAR is located at the James Ax Observatory, at an altitude of 5200 meters on Cerro Toco in the Atacama desert in Chile and achieved first light in January 2012. This site was chosen for its dry, stable weather, with precipitable water vapor (PWV) less than $1.5 \mathrm{~mm}$ for over $50 \%$ of the year. This corresponds to a sky brightness in the POLARBEAR design band of $15 K_{R J}$ at an elevation angle of 60 degrees.

Calibration is key to understanding the instrument's performance, and POLARBEAR uses both hardware and astrophysical calibration sources. Calibration data for relative detector response is taken every hour during observations. The relative detector response is measured using a 3 minute observation of a variable frequency chopped thermal source located behind an aperture in the secondary mirror, with an effective temperature of $0.03 \mathrm{~K}$. Relative detector response is also measured using fast elevation scans that vary detector response due to the changing line-of-sight air mass, spanning 3 degrees of elevation and approximately 0.5 Kelvin of sky temperature modulation. The relative detector response is used to calculate a differential timestream for the response of the two orthogonally oriented detectors within one pixel. This timestream can be used to measure the $\mathrm{Q}$ or U Stokes parameters while suppressing the unpolarized atmospheric signal.

The large fraction of the sky available to POLARBEAR means that many astrophysical sources can be observed. Planets like Jupiter and Saturn are bright 


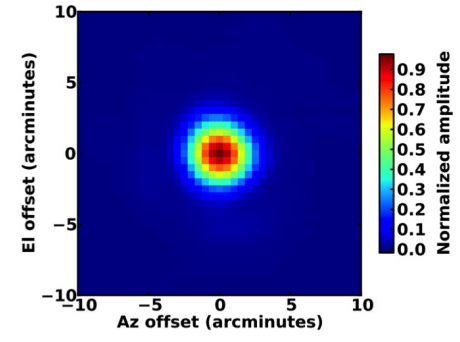

(a)

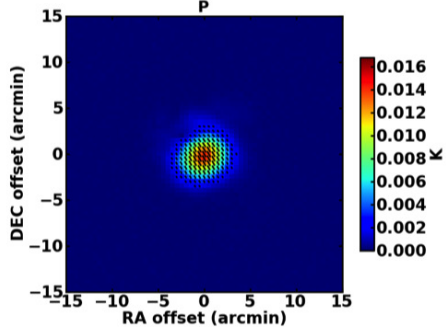

(b)

Fig. 3: (a) Coadded angular response of observations of Saturn (b) Intensity and polarization map of Taurus A. (Color figure online)

point sources, so observations of these planets map the structure of the beams. Each detector beam's size, ellipticity, and offset from boresite can be measured using planets. Figure 3 a shows a coadded map made from active detectors observations of Saturn. The measured median FWHM beam size is 3.5 arcminutes, with a median ellipticity of $0.05^{6}$. Planet observations can also be used to determine detector yield and NET, which vary with atmospheric conditions. The total number of active detectors is 1015, the typical operating yield during observations is about 900 detectors, and the median detector NET is $550 \mu \mathrm{K} \sqrt{s}$. Another important astrophysical calibrator for POLARBEAR is Taurus A, a supernova remnant in the Crab Nebula. Taurus A has a well-known polarization angle, caused by synchrotron emission ${ }^{13}$, and is used to calibrate detector polarization angles. Figure $3 \mathrm{~b}$ shows a coadded temperature map of Tau A with the resulting polarization $P=\sqrt{Q^{2}+U^{2}}$ and polarization angle of the source.

The POLARBEAR collaboration is not examining B-mode polarization results until the instrumental and data quality studies using other indicators are complete. Shown in Figure 4 is the E-mode polarization for one of three sky patches observed by POLARBEAR. A preliminary map depth for this patch has been calculated, which is $5 \mu \mathrm{K}_{C M B}-$ arcminute for polarization.

\section{Future plans}

POLARBEAR is in its second season of observations, and the POLARBEAR collaboration is currently analyzing the first season of data. Upgrades to the project are also underway, with two additional telescopes, known as the Simons Array, and the next-generation dichroic receiver, POLARBEAR-2 ${ }^{14}$ under construction.

Acknowledgements POLARBEAR is funded by the National Science Foundation under grant AST-0618398. Antenna-coupled bolometer development at Berkeley is funded by NASA under grant NNG06GJ08G. The McGill authors acknowledge funding from the Natural Sciences and Engineering Research Council and Canadian Institute for Advanced Research. The KEK authors are supported by MEXT KAKENHI Grant Number 21111002. The Chilean site is located in the Parque Astronomico Atacama in agreement with the Comisión Nacional de Investigación Científica y Tecnológica de Chile. The Simons Array is funded by the Simons Foundation. 


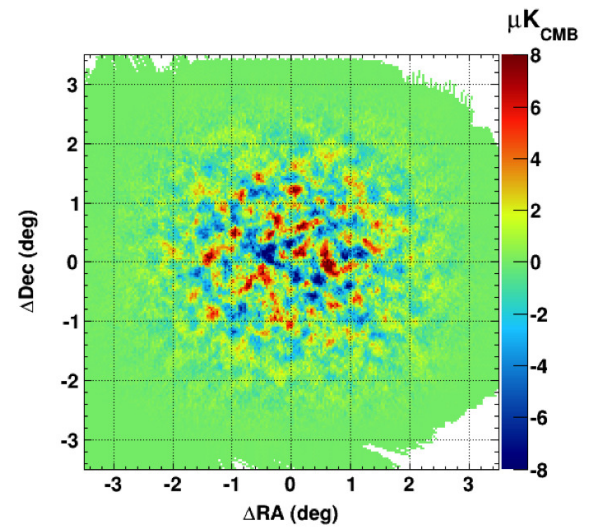

Fig. 4: Preliminary first season E-mode polarization map for one of three observation patches, with approximately 1700 hours of observation time. (Color figure online)

\section{References}

1. U. Seljak, and M. Zaldarriaga, Phys. Rev. Lett. 78, 2054 (1997).

2. J. Kovac et al., Nature 20, $772-787$ (2002).

3. Bennet et al., ApJS 20820 (2013).

4. D. Hanson et al., Phys. Rev. Lett. 111, 141301 (2013).

5. C. H. Chiang et al., Astrophys. J. 711, 1123-1140 (2010).

6. Z. Kermish et al., Proc. SPIE $\mathbf{8 4 5 2}$ (2012)

7. K. Arnold et al., Proc. SPIE 8452 (2012)

8. M. Myers et al., J Low Temp Phys 151: 464-470 (2008)

9. H. Tran et al, Applied Optics 47(2), pp. 103109, 2008.

10. A. T. Lee et al., American Institute of Physics Conference Series (2009).

11. D. Filipovic et al., IEEE Trans. on Micr. Theory and Tech., 41(10) (1993)

12. M. A. Dobbs et al., Rev. Sci. Instrum. 83, 073113 (2012)

13. J. Aumont et al., $A \& A 13834$ (2010)

14. A. Suzuki et al., These proceedings (2013) 